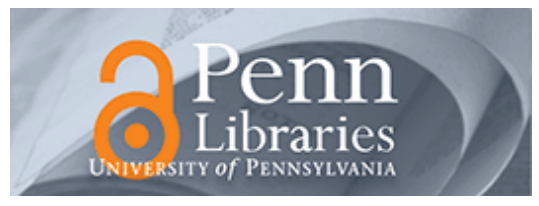

University of Pennsylvania

ScholarlyCommons

Statistics Papers

Wharton Faculty Research

2011

\title{
On the Fermat-Weber Point of a Polygonal Chain and Its Generalizations
}

Bhaswar B. Bhattacharya

University of Pennsylvania

Follow this and additional works at: https://repository.upenn.edu/statistics_papers

Part of the Applied Mathematics Commons, Business Administration, Management, and Operations Commons, Business Analytics Commons, Management Sciences and Quantitative Methods Commons, Mathematics Commons, and the Statistics and Probability Commons

\section{Recommended Citation}

Bhattacharya, B. B. (2011). On the Fermat-Weber Point of a Polygonal Chain and Its Generalizations. Fundamenta Informaticae, 107 (4), 331-343. http://dx.doi.org/10.3233/FI-2011-406

This paper is posted at ScholarlyCommons. https://repository.upenn.edu/statistics_papers/653

For more information, please contact repository@pobox.upenn.edu. 


\title{
On the Fermat-Weber Point of a Polygonal Chain and Its Generalizations
}

\begin{abstract}
In this paper, we study the properties of the Fermat-Weber point for a set of fixed points, whose arrangement coincides with the vertices of a regular polygonal chain. A $k$-chain of a regular $n$-gon is the segment of the boundary of the regular $n$-gon formed by a set of $k(\leq n)$ consecutive vertices of the regular $n$-gon. We show that for every odd positive integer $k$, there exists an integer $N(k)$, such that the Fermat-Weber point of a set of $k$ fixed points lying on the vertices a $k$-chain of a $n$-gon coincides with a vertex of the chain whenever $n \geq N(k)$. We also show that $\lceil\pi m(m+1)-\pi 2 / 4\rceil \leq N(k) \leq\lfloor\pi m(m+1)+1\rfloor$, where $k(=2 m+1)$ is any odd positive integer. We then extend this result to a more general family of point set, and give an $O(h k \log k)$ time algorithm for determining whether a given set of $k$ points, having $h$ points on the convex hull, belongs to such a family.
\end{abstract}

\section{Keywords}

computational geometry, facility location, Fermat-Weber problem, optimization, polygons

Disciplines

Applied Mathematics | Business | Business Administration, Management, and Operations | Business Analytics | Management Sciences and Quantitative Methods | Mathematics | Statistics and Probability 


\title{
On the Fermat-Weber Point of a Polygonal Chain and its Generalizations*
}

\author{
Bhaswar B. Bhattacharya \\ Department of Statistics, Stanford University \\ bhaswar. bhattacharya@gmail.com
}

\begin{abstract}
In this paper, we study the properties of the Fermat-Weber point for a set of fixed points, whose arrangement coincides with the vertices of a regular polygonal chain. A $k$-chain of a regular $n$-gon is the segment of the boundary of the regular $n$-gon formed by a set of $k(\leq n)$ consecutive vertices of the regular $n$-gon. We show that for every odd positive integer $k$, there exists an integer $N(k)$, such that the Fermat-Weber point of a set of $k$ fixed points lying on the vertices a $k$-chain of a $n$-gon coincides with a vertex of the chain whenever $n \geq N(k)$. We also show that $\left\lceil\pi m(m+1)-\pi^{2} / 4\right\rceil \leq N(k) \leq\lfloor\pi m(m+1)+1\rfloor$, where $k(=2 m+1)$ is any odd positive integer. We then extend this result to a more general family of point set, and give an $O(h k \log k)$ time algorithm for determining whether a given set of $k$ points, having $h$ points on the convex hull, belongs to such a family.
\end{abstract}

Keywords: Computational geometry, Facility location, Fermat-Weber Problem, Optimization, Polygons.

\section{Introduction}

The Fermat-Weber point $\mathcal{W}(S)$ of a set $S$ of $n$ points $\left\{p_{1}, p_{2}, \ldots, p_{n}\right\}$ in $\mathbb{R}^{d}$ is the point $p$ which minimizes the sum $\sum_{i=1}^{n} d\left(p, p_{i}\right)$ [10]19]24] where $d(\alpha, \beta)$ denotes the Euclidean distance between the two points $\alpha$ and $\beta$. The origin of this problem is attributed to the great Pierre de Fermat (1601-1665) who, four centuries ago, asked to find a point which minimizes the sum of Euclidean distances to three fixed points in the plane. Around the year 1640, Evangelista Torricelli (1608-1647) devised a geometrical construction for this problem. He showed that the point minimizing the sum of distances from the three fixed points is the point inside the triangle determined by the three fixed points, at which every side of the triangle subtends an angle of $2 \pi / 3$. This, however, is true only when all the interior angles of the triangle are at most $2 \pi / 3$. The so-called complementary problem where one angle of the triangle can be greater than $2 \pi / 3$ first appeared in Courant and Robbins' famous book What is mathematics? The solution of the complementary problem, which states that the optimum always point coincides with the obtuse vertex of the triangle, was correctly proved later by Krarup and Vajda [18. The solution of the Fermat-Weber problem with weights associated with each of the three points is also known. The solution for positive weights can be found in the book by Yaglom [27. Jalal et al. [16] completely describes the solution when negative weights are also allowed.

The sum of Euclidean distances to four fixed points in the plane is minimized at the point of intersection of the diagonals, when the fixed points form a convex quadrangle.

\footnotetext{
* This work was done when the author was an undergraduate student at the Indian Statistical Institute, Kolkata, India, with financial support from the Department of Science and Technology (DST), Govt. of. India, under the KVPY fellowship award.
} 
Otherwise, the sum is minimized at the fixed point which coincides with the concave corner of the quadrangle formed by the four fixed points. The convex case was first solved by Fagnano [13, but the origin of the solution for the other case remains unknown. Recently Plastria 21] gave new proofs for both the cases and generalized these results to general metrics and norms.

Bajaj [3] showed that even for 5 points, the coordinates of the Fermat-Weber point may not be representable even if we allow radicals, and that it is impossible to construct an optimal solution by means of a ruler and a compass. There are only a few patterns where the location Fermat-Weber point can be determined exactly. A point set $S$ is said to form an equiangular configuration if there exists a point $c \notin S$ and an ordering of the points in $S$ such that each two adjacent points form an angle of $2 \pi / n$ with respect to $c$. The Fermat-Weber point of an equiangular configuration is the point $c$. Anderegg et al. 2] presented a linear time algorithm to identify whether a given set of points is in equiangular configuration.

However, it is difficult to exactly determine the Fermat-Weber point of a set of fixed points unless it has a highly symmetric arrangement. As a matter of fact, it is hard to find the Fermat-Weber point even if all fixed points lie on a circle 9].

Haldane [15] proved that the Fermat-Weber point is unique for any point set in $\mathbb{R}^{d}(d \geq 2)$, unless the points all lie on a single straight line. However, no algorithm for computing the exact solution to the Fermat-Weber problem is known. The most famous of all existing algorithms is the iterative algorithm due to Weiszfeld [25]. Later Vardi and Zhang [23] gave a simple modification of this algorithm for solving the Fermat-Weber location problem in $\mathbb{R}^{d}$ with extensions to more general cost functions. Bose et al. 4] derived $\epsilon$-approximation algorithms for the Fermat-Weber problem in any fixed dimension, using geometric data structures.

The relevance of the Fermat-Weber problem in location science was first envisaged in 1909 by Alfred Weber [24, when he studied the locational optimization of a firm in a region. Thereafter, the problem of minimizing the sum of distances from a given set of fixed points is referred to as the Fermat-Weber problem. Since then the min-sum criteria has been as an optimization criterion in several facility location problems and extensive research has been done on them over the years ([10], 26]). Recently, Burkard et al. [5] introduced the inverse Fermat-Weber problem, where a set of $n$ points in the plane with nonnegative weights is given, and the objective is to change the weights at minimum cost such that a prespecified point in the plane becomes the Fermat-Weber point. Carmi et al. [6] studied the FermatWeber point of planar convex objects. Their bounds were improved by Abu-Affash and Katz [1], and later by Dumitrescu et al. [11. In a related paper, Dumitrescu et al. [12] studied minimum length stars and Steiner stars of planar point sets. Apart from its relevance in facility location, the Fermat-Weber problem also finds importance in statistics, especially in the definition of medians [14] and quantiles [8] of multivariate data.

In this paper, we study the properties of the Fermat-Weber point for a set of fixed points, whose arrangement coincides with the vertices of a regular polygonal chain. A $k$-chain of a regular $n$-gon (or a regular polygonal chain of length $k$ ) is the segment of the boundary of the regular $n$-gon formed by a set of $k(\leq n)$ consecutive vertices of the regular $n$-gon. A $k$-chain of a regular $n$-gon will be denoted by $C_{n}(k) . \mathcal{W}\left(C_{n}(k)\right)$ denotes the Fermat-Weber point of the set of $k$ fixed points which coincides with the vertices of the chain $C_{n}(k)$. A chain is said to be empty if it has no vertices, that is, $k=0$. In Section 2 we study some of the properties of $\mathcal{W}\left(C_{n}(k)\right)$. Observe that when a vertex of a chain $C_{n}(k)$ is deleted, we 
get two smaller chains of the same regular $n$-gon, one of which may be empty. Now, if $k$ is an odd integer, there exists a vertex of the chain $C_{n}(k)$, which when deleted gives two identical smaller chains. We call this vertex the root vertex of the chain. We show that for every odd positive integer $k$, there exists an integer $N(k)$ such that $\mathcal{W}\left(C_{n}(k)\right)$, coincides with the root vertex of $C_{n}(k)$, whenever $n \geq N(k)$. This can be thought of as an extension of Courant and Robbins' complementary problem on triangles. In Section 3 we prove that $\left\lceil\pi m(m+1)-\pi^{2} / 4\right\rceil \leq N(k) \leq\lceil\pi m(m+1)+1\rceil$, where $k=2 m+1(m \geq 1)$, is any odd positive integer. In Section 4, we extend this result to a more general family of point set. We also present an $O(h k \log k)$ time algorithm for determining whether a given set of $k$ points, having $h$ points on the convex hull, belongs to such a family. Finally, in Section 5 we summarize our work and give some directions for future work.

\section{Fermat-Weber Point of Polygonal Chains}

In this section we prove various properties of the Fermat-Weber point of a set of fixed points lying on the vertices of a polygonal chain. We denote by $\mathcal{V}\left(C_{n}(k)\right)$ the set of vertices of the chain $C_{n}(k)$. In the following we shall assume that the vertices of the chain $C_{n}(k)$ lie on the circumference of a unit circle with center at the point $o$, because the Fermat-Weber point of a set of fixed points remains invariant under uniform scaling.

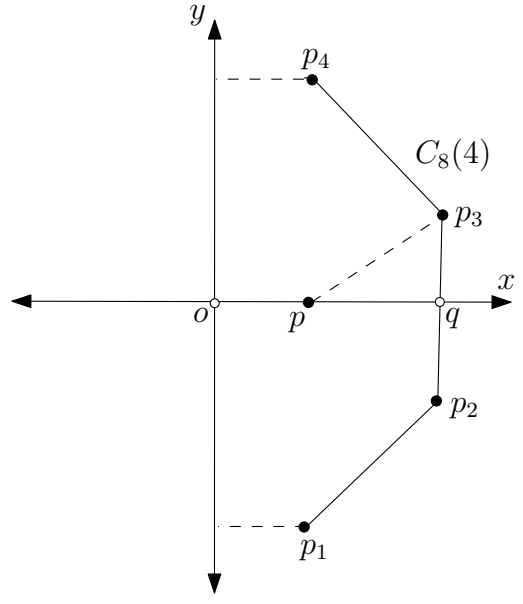

(a)

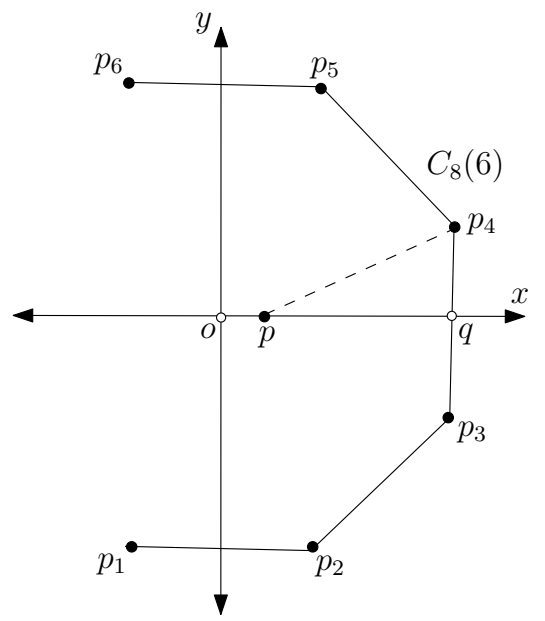

(b)

Fig. 1. Even Polygonal Chains: (a) $C_{8}(4)$ : 4-chain of a regular 8-gon, (b) $C_{8}(6)$ : 6-chain of a regular 8-gon.

Now, depending on whether $k$ is even or odd we have the following two cases:

Case 1: $k=2 m, m \geq 1$. Let $\mathcal{V}\left(C_{n}(k)\right)=\left\{p_{1}, p_{2}, \ldots, p_{k}\right\}$ be the vertices of the chain taken in the counter-clockwise direction starting with the lowermost vertex of the chain, as shown in Figure 1. Observe that the chain is symmetric about the line oq, where $o$ is the circumcenter of $\mathcal{V}\left(C_{n}(k)\right)$ and $q$ is the midpoint of the line segment $p_{m} p_{m+1}$. We call the line $o q$ the line of symmetry of the chain. The uniqueness of the Fermat-Weber point 
now implies that $\mathcal{W}\left(C_{n}(k)\right)$ must lie on this line. Consider the rectangular coordinate system with origin at the point $o$ and $o q$ as the horizontal axis. Let $p:=(x, 0), x>0$, be a point on the horizontal axis. The sum of distances from the point $p$ to the vertices of $C_{n}(k)$ is then given by

$$
\psi(k, n, x)=\sum_{p_{i} \in \mathcal{V}\left(C_{n}(k)\right)} d\left(x, p_{i}\right)=\sum_{i=1}^{m} 2 \sqrt{x^{2}-2 \mu_{i}(n) x+1} .
$$

where $\mu_{i}(n)=\cos ((2 i-1) \pi / n)$. The point $p_{0}$ on the line segment $\overline{o q}$, where $\psi(k, n, x)$ is minimized is the location of $\mathcal{W}\left(C_{n}(k)\right)$. We denote by $\left|\mathcal{W}\left(C_{n}(k)\right)\right|$ the distance of the point $p_{0}$ from the origin $o$.

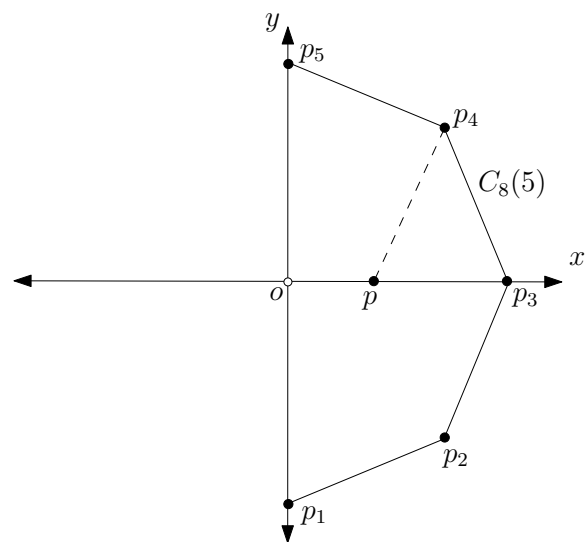

(a)

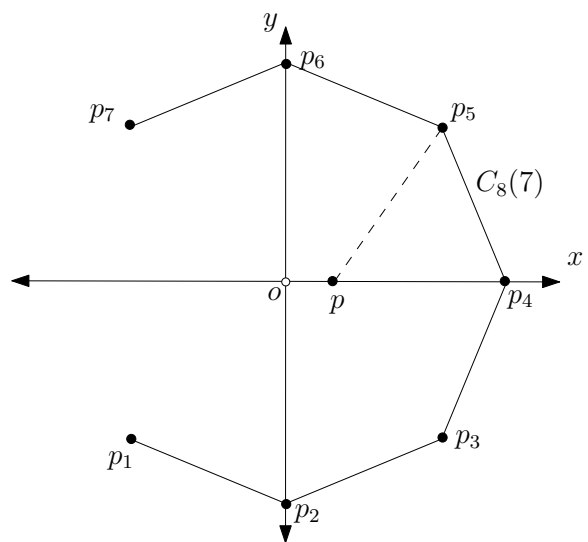

(b)

Fig. 2. Odd Polygonal Chains: (a) $C_{8}(5)$ : 5 -chain of a regular 8-gon, (b) $C_{8}(7)$ : 7-chain of a regular 8-gon.

Case 2: $k=2 m+1, m \geq 1$. Let $\mathcal{V}\left(C_{n}(k)\right)=\left\{p_{1}, p_{2}, \ldots, p_{k}\right\}$ be the vertices of the chain taken in the counter-clockwise direction starting with the lowermost vertex of the chain, as shown in Figure 2. In this case, the chain is symmetric about the line $o p_{m+1}$, which then implies that $\mathcal{W}\left(C_{n}(k)\right)$ must lie on this line. We call the line $o p_{m+1}$ the line of symmetry of the chain, and the vertex $p_{m+1}$ the root vertex of the chain. Consider the rectangular coordinate system with the circumcenter $o$ of $\mathcal{V}\left(C_{n}(k)\right)$ as the origin and the line $o p_{m+1}$ as the horizontal axis. If $p:=(x, 0), x \in[0,1]$, is a point on the $x$-axis, sum of distances from $p$ to the vertices of the $C_{n}(k)$ is given by

$$
\psi(k, n, x)=\sum_{p_{i} \in \mathcal{V}\left(C_{n}(k)\right)} d\left(x, p_{i}\right)=1-x+\sum_{i=1}^{m} 2 \sqrt{x^{2}-2 x \lambda_{i}(n)+1}
$$

where $\lambda_{i}(n)=\cos (2 i \pi / n)$. As before, the point $p_{0}$ on the line segment $\overline{o p_{m+1}}$ where $\psi(k, n, x)$ is minimized is the location of $\mathcal{W}\left(C_{n}(k)\right)$. We denote by $\mid \mathcal{W}\left(C_{n}(k) \mid\right.$ the distance of the point $p_{0}$ from the origin $o$.

Henceforth, we shall always consider the coordinate system described above while doing any computation with polygonal chains. 
We begin with the following well known observation about the function $\psi(k, n, x)$, which will be repeatedly used in the proofs of the subsequent results.

Observation 1 For positive integers $n, k$ such that $n \geq k$ and $k \geq 3$, the objective function $\psi(k, n, x)$ is strictly convex for all $x \in[0,1]$.

Proof. The double derivative of $\psi(k, n, x)$ for all $x \in[0,1]$ is

$$
\psi_{x}^{\prime \prime}(k, n, x)=\frac{\partial^{2}}{\partial x^{2}} \psi(k, n, x)=\sum_{\mathcal{V}\left(C_{n}(k)\right)} \frac{1-\beta_{i}^{2}(n)}{\left[x^{2}-2 \beta_{i}(n) x+1\right]^{3 / 2}} .
$$

where $\beta_{i}(n)=\lambda_{i}(n)$ when $k$ is odd and $\beta_{i}(n)=\mu_{i}(n)$ when $k$ is even. The result now follows from the fact that $\psi_{x}^{\prime \prime}(k, n, x)$ is positive for all $x \in[0,1]$.

Now, we fix a value of $k$ and study the variation in the Fermat-Weber point of $C_{n}(k)$ as $n$ varies. Table 1 lists the location of the Fermat-Weber points and the corresponding values of the objective function for $C_{n}(4)$, for all $n \leq 18$ varies. Table 2 lists the same for the chain $C_{n}(5)$. These values were obtained by numerically solving the equation $\frac{\partial}{\partial x} \psi(k, n, x)=0$ using Mathematica 4.0.

Table 1. $\mathcal{W}\left(C_{n}(4)\right)$ for $n \leq 18$

\begin{tabular}{|c|c|c|}
\hline$n$ & $\psi\left(4, n,\left|\mathcal{W}\left(C_{n}(4)\right)\right|\right)$ & $\mid \mathcal{W}\left(C_{n}(4) \mid\right.$ \\
\hline 4 & 4.00000 & 0.000000 \\
\hline 5 & 3.80423 & 0.381966 \\
\hline 6 & 3.4641 & 0.577352 \\
\hline 7 & 3.12733 & 0.692021 \\
\hline 8 & 2.82843 & 0.765369 \\
\hline 9 & 2.57115 & 0.815208 \\
\hline 10 & 2.35114 & 0.85065 \\
\hline 11 & 2.16256 & 0.876769 \\
\hline 12 & 2.00000 & 0.896575 \\
\hline 13 & 1.85889 & 0.911956 \\
\hline 14 & 1.73553 & 0.924139 \\
\hline 15 & 1.62695 & 0.933955 \\
\hline 16 & 1.53073 & 0.941979 \\
\hline 17 & 1.44497 & 0.948624 \\
\hline 18 & 1.36808 & 0.954189 \\
\hline
\end{tabular}

Table 2. $\mathcal{W}\left(C_{n}(5)\right)$ for $n \leq 19$

\begin{tabular}{|c|c|c|}
\hline$n$ & $\psi\left(5, n,\left|\mathcal{W}\left(C_{n}(5)\right)\right|\right)$ & $\mathcal{W}\left(C_{n}(5) \mid\right.$ \\
\hline 5 & 5.00000 & 0.000000 \\
\hline 6 & 4.83419 & 0.330454 \\
\hline 7 & 4.50791 & 0.534378 \\
\hline 8 & 4.15356 & 0.667873 \\
\hline 9 & 3.81793 & 0.759008 \\
\hline 10 & 3.51502 & 0.82332 \\
\hline 11 & 3.2466 & 0.869949 \\
\hline 12 & 3.01013 & 0.904536 \\
\hline 13 & 2.80181 & 0.930659 \\
\hline 14 & 2.61783 & 0.950717 \\
\hline 15 & 2.45470 & 0.966323 \\
\hline 16 & 2.30942 & 0.978603 \\
\hline 17 & 2.17944 & 0.98836 \\
\hline 18 & 2.06261 & 0.996175 \\
\hline 19 & 1.95718 & 1.00000 \\
\hline
\end{tabular}

From the values listed these two tables it can be observed that the distance of $\mathcal{W}\left(C_{n}(k)\right)$ from the circumcenter $o$ increases as $n$ increases. Hence, we have the following observation:

Observation 2 If $n_{1}, n_{2}, k$ are positive integers, such that $k \leq n_{1}<n_{2}$, then

(i) $\psi\left(k, n_{1}, \mid \mathcal{W}\left(C_{n_{1}}(k) \mid\right)>\psi\left(k, n_{2}, \mid \mathcal{W}\left(C_{n_{2}}(k) \mid\right)\right.\right.$,

(ii) $\mid \mathcal{W}\left(C_{n_{1}}(k)|<| \mathcal{W}\left(C_{n_{2}}(k)\right) \mid\right.$, whenever $\mid \mathcal{W}\left(C_{n_{1}}(k) \mid<1\right.$ and $\mid \mathcal{W}\left(C_{n_{2}}(k) \mid<1\right.$.

Proof. Since $\cos \theta$ is a decreasing function for $\theta \in[0, \pi]$, it follows that if $n_{1}<n_{2}$, then $\mu_{i}\left(n_{1}\right)<\mu_{i}\left(n_{2}\right)$ and $\lambda_{i}\left(n_{1}\right)<\lambda_{i}\left(n_{2}\right)$, for every fixed $i$. Equations (11) and (2) now imply that if $n_{1}<n_{2}$, then $\psi\left(k, n_{1}, x\right)>\psi\left(k, n_{2}, x\right)$ for all $x \in[0,1]$. In particular,

$$
\psi\left(k, n_{1}, \mid \mathcal{W}\left(C_{n_{1}}(k) \mid\right) \geq \psi\left(k, n_{2}, \mid \mathcal{W}\left(C_{n_{1}}(k) \mid\right)>\psi\left(k, n_{2}, \mid \mathcal{W}\left(C_{n_{2}}(k) \mid\right),\right.\right.\right.
$$


which proves the first part.

Next, let $\psi^{\prime}(k, n, x)=\frac{\partial}{\partial x} \psi(k, n, x)$. Now, since $\mid \mathcal{W}\left(C_{n_{1}}(k) \mid<1\right.$ and $\mid \mathcal{W}\left(C_{n_{2}}(k) \mid<1\right.$, the minimum of the function $\psi$ lies in the interval $[0,1)$. This implies that $\psi^{\prime}\left(k, n_{1},\left|\mathcal{W}\left(C_{n_{1}}(k)\right)\right|\right)=$ $\psi^{\prime}\left(k, n_{2},\left|\mathcal{W}\left(C_{n_{2}}(k)\right)\right|\right)=0$. It is easy to see that for any fixed values of $k$ and $x, \psi^{\prime}(k, n, x)$ is a decreasing function of of $n$. Therefore, $\psi^{\prime}\left(k, n_{2},\left|\mathcal{W}\left(C_{n_{1}}(k)\right)\right|\right)<\psi^{\prime}\left(k, n_{1},\left|\mathcal{W}\left(C_{n_{1}}(k)\right)\right|\right)=0$, and $\psi^{\prime}\left(k, n_{2},\left|\mathcal{W}\left(C_{n_{2}}(k)\right)\right|\right)=0$. Now, since $\psi(k, n, x)$ is convex for all $x \in[0,1]$, the derivative $\psi^{\prime}(k, n, x)$ must be non-decreasing in $x$ in the interval $[0,1]$. This implies that $\left|\mathcal{W}\left(C_{n_{1}}(k)\right)\right|<\left|\mathcal{W}\left(C_{n_{2}}(k)\right)\right|$.

From the solution of the complementary problem [18] we already know that the FermatWeber point of a 3 -chain coincides with the root vertex of $C_{n}(3)$ if $n \geq 6$. This fact and the values in Table 2 motivate the formulation of the following lemma.

Lemma 1. For every odd positive integer $k=2 m+1(m \geq 1), \mathcal{W}\left(C_{n}(k)\right)$ coincides with the root vertex of the chain $C_{n}(k)$, if and only if $2 \sum_{i=1}^{m} \sin (i \pi / n)-1 \leq 0$.

Proof. $(\Rightarrow)$ If $\mathcal{W}\left(C_{n}(k)\right)$ coincides with the root vertex of the chain $C_{n}(k)$, the objective function $\psi(k, n, x)$ is minimized at the point $x=1$, for all $x \in[0,1]$. This implies that $\psi(k, n, x)$ must be non-increasing on the interval $[0,1]$, because $\psi(k, n, x)$ is convex on the interval $[0,1]$. Therefore,

$$
\psi^{\prime}(k, n, x)=\frac{\partial}{\partial x} \psi(k, n, x)=2 \sum_{i=1}^{m} \frac{x-\lambda_{i}(n)}{\sqrt{x^{2}-2 x \lambda_{i}(n)+1}}-1 \leq 0 \quad \forall x \in[0,1] .
$$

where $\lambda_{i}(n)=\cos 2(i \pi / n)$. This implies that $\psi^{\prime}(k, n, 1)=2 \sum_{i=1}^{m} \sin (i \pi / n)-1 \leq 0$.

$(\Leftarrow)$ We know that $\psi^{\prime}(k, n, 1)=2 \sum_{i=1}^{m} \sin (i \pi / n)-1 \leq 0$. This implies that $\psi^{\prime}(k, n, x) \leq 0$ for all $x \in[0,1]$, because $\psi^{\prime}(k, n, x)$ is non-decreasing in $x$ on the interval $[0,1]$, by the convexity of $\psi$ on $[0,1]$. Therefore, $\psi(k, n, x)$ is non-increasing on the interval $[0,1]$ and is minimized at the endpoint $x=1$, that is, $\mathcal{W}\left(C_{n}(k)\right)$ coincides with the root vertex of the chain $C_{n}(k)$.

Let us denote $f(n)=2 \sum_{i=1}^{m} \sin (i \pi / n)-1$. Observe that as $n$ increases, $f(n)$ decreases, and if $n$ is large enough $f(n)$ becomes non-positive, since $\lim _{n \rightarrow \infty} f(n)=-1$. Therefore, the condition $f(n) \leq 0$ holds whenever $n$ is sufficiently large, and the following theorem is immediate.

Theorem 1. For every odd positive integer $k=2 m+1(m \geq 1)$, there exists a smallest integer $N(k)$ such that for all $n \geq N(k), \mathcal{W}\left(C_{n}(k)\right)$ coincides with the root vertex of $C_{n}(k)$. Moreover, $N(k)=\min \left\{t \in \mathbb{N}: 2 \sum_{i=1}^{m} \sin (i \pi / t)-1 \leq 0\right\}$.

Theorem 1 can be viewed as an extension of the complementary problem of Courant and Robbins. It follows from the result of the complementary problem that $N(3)=6$, since the interior angles of a regular hexagon are $2 \pi / 3$. Theorem 1 asserts that $N(k)$ can be determined by checking the sign of $2 \sum_{i=1}^{m} \sin (i \pi / n)-1$ at all the integers till the first time it becomes non-positive. The values $N(k)$ for some small values of $k$ are shown in Table 3 ,

Note on Even Chains : Consider a $k$-chain $C_{n}(k)$, where $k=2 m(m \geq 1)$ is an even integer. Let $q$ be the point where the line of symmetry of $C_{n}(k)$ intersects the boundary of the chain. 
Table 3. $N(k)$ for some small values of $k$

\begin{tabular}{|c|c|c|c|c|c|c|c|c|c|c|c|c|}
\hline$k$ & 3 & 5 & 7 & 9 & 11 & 13 & 15 & 17 & 19 & 21 & 23 & 25 \\
\hline$N(k)$ & 6 & 19 & 38 & 63 & 94 & 132 & 176 & 226 & 283 & 346 & 415 & 490 \\
\hline
\end{tabular}

As in the case of odd chains, one might conjecture that $\mathcal{W}\left(C_{n}(k)\right)$ coincides with $q$ if $n$ is sufficiently large. This, however, is not true. The coordinates of the point $q$ are $\left(\mu_{1}(n), 0\right)$, where $\mu_{1}(n)=\cos (\pi / n)$. It now follows from Equation (11) that

$$
\psi^{\prime}(k, n, x)=\frac{\partial}{\partial x} \psi(k, n, x)=2 \sum_{i=1}^{m} \frac{x-\mu_{i}(n)}{\sqrt{x^{2}-2 x \mu_{i}(n)+1}} \forall x \in\left[0, \mu_{1}(n)\right] .
$$

Note that for every fixed $n, \mu_{i}(n) \leq \pi$, for all $i \in\{1,2, \ldots, m\}$. Since, $\cos \theta$ is a decreasing function for $\theta \in[0, \pi]$, we have $\mu_{1}(n)>\mu_{i}(n)$, for $i \in\{2, \ldots, m\}$. Equation (3) now implies that $\psi^{\prime}\left(k, n, \mu_{1}(n)\right)>0$, for every fixed $n$. From the convexity of the function $\psi$ proved in Observation 1, we now conclude that $\left|\mathcal{W}\left(C_{n}(k)\right)\right|<\mu_{1}(n)$, for every fixed $n$. Therefore, as $n$ increases the Fermat-Weber point of the even chain $C_{n}(k)$ gradually approaches the point $q$, but it never actually coincides with $q$ for any finite value of $n$. This implies that $N(2 m)=\infty$, for $m \geq 2$, and illustrates the impossibility of a result analogous to Theorem 1 for even $k$-chains.

It is in fact the root vertex, which dominates the location of $\mathcal{W}\left(C_{n}(k)\right)$, when $k$ is odd, by pulling it towards itself as the value of $n$ increases.

\section{Determination of $N(k)$}

In this section we determine bounds on the number $N(k)$ and propose an algorithm for determining it.

At first, we have the following observation:

Observation 3 For every odd positive integer $k(=2 m+1) \geq 3$, we have $N(k) \geq m(m+1)$.

Proof. Observe that $f(m(m+1))=2 \sum_{i=1}^{m} \sin (i \pi /(m(m+1)))-1$. Now, using the fact that for $\theta \in[0, \pi], \sin \theta \geq \theta-\theta^{3} / 6$, we get

$$
\begin{aligned}
f(m(m+1)) & =2 \sum_{i=1}^{m} \sin \frac{i \pi}{m(m+1)}-1 \\
& \geq 2 \sum_{i=1}^{m} \frac{i \pi}{m(m+1)}-2 \sum_{i=1}^{m} \frac{i^{3} \pi^{3}}{6 m^{3}(m+1)^{3}}-1 \\
& \geq \pi-\frac{\pi^{3}}{12 m(m+1)}-1
\end{aligned}
$$

where the last equation follows from the fact that $\sum_{i=1}^{m} i=m(m+1) / 2$ and $\sum_{i=1}^{m} i^{3}=$ $m^{2}(m+1)^{2} / 4$. Now, since for all $m \geq 1, m(m+1) \geq 2$ we get from Equation (44), $f(m(m+$ 1)) $\geq \pi-\frac{\pi^{3}}{24}-1>0$. This prove that $N(k) \geq m(m+1)$ for $k=2 m+1, m \geq 1$. 
Using this observation, we now prove the following bounds on $N(k)$.

Theorem 2. For every odd positive integer $k(=2 m+1), m \geq 1$, we have

$$
\left\lceil\pi m(m+1)-\pi^{2} / 4\right\rceil \leq N(k) \leq\lfloor\pi m(m+1)+1\rfloor .
$$

Proof. Let $C_{n}(k)$ be a regular polygonal chain of length $k$, where $k=2 m+1, m \geq 1$. From Theorem 1 we have $N(k)=\min \left\{t \in \mathbb{N}: 2 \sum_{i=1}^{m} \sin (i \pi / t)-1 \leq 0\right\}$. Since $N(k)$ is the smallest integer of this set, we must have, $2 \sum_{i=1}^{m} \sin (i \pi /(N(k)-1))-1>0$. Observe that for every $i \in\{1,2, \ldots, m\}$, the function $\sin (i \pi /(N(k)-1))$ is continuous and differentiable in the interval $(0, i \pi /(N(k)-1))$. Hence, by the Mean Value Theorem, for every $i \in\{1,2, \ldots, m\}$ there exists $\theta_{i}^{*} \in(0, i \pi /(N(k)-1))$ such that

$$
2 \sum_{i=1}^{m} \sin \frac{i \pi}{N(k)-1}-1=2 \sum_{i=1}^{m} \frac{i \pi}{N(k)-1} \cos \theta_{i}^{*}-1>0 .
$$

This implies, $N(k)<2 \pi \sum_{i=1}^{m} i \cos \theta_{i}^{*}+1 \leq 2 \pi \sum_{i=1}^{m} i+1=\pi m(m+1)+1$. This proves that for $m \geq 1$, we have $N(k) \leq\lfloor\pi m(m+1)+1\rfloor$.

To prove the lower bound, observe that $2 \sum_{i=1}^{m} \sin i \pi / N(k)-1 \leq 0$. By the Mean Value Theorem, for every $i \in\{1,2, \ldots, m\}$ there exists $\theta_{i} \in(0, i \pi / N(k))$ such that

$$
2 \sum_{i=1}^{m} \sin \frac{i \pi}{N(k)}-1=2 \sum_{i=1}^{m} \frac{i \pi}{N(k)} \cos \theta_{i}-1 \leq 0 .
$$

This implies that $N(k) \geq 2 \pi \sum_{i=1}^{m} i \cos \theta_{i}$. Using the inequality $\cos \theta_{i} \geq 1-\theta_{i}^{2} / 2$, for all $i \in\{1,2, \ldots, m\}$, and the fact that $\theta_{i} \in(0, i \pi / N(k))$, we get $\cos \theta_{i} \geq 1-\frac{i^{2} \pi^{2}}{2(N(k))^{2}}$. Now, since from Observation 3 , we know that $N(k) \geq m(m+1)$, we get $\cos \theta_{i} \geq 1-\frac{i^{2} \pi^{2}}{2 m^{2}(m+1)^{2}}$, for all $i \in\{1,2, \ldots, m\}$. Therefore,

$$
\begin{aligned}
N(k) \geq 2 \pi \sum_{i=1}^{m} i \cos \theta_{i} & \geq 2 \pi \sum_{i=1}^{m} i\left(1-\frac{i^{2} \pi^{2}}{2 m^{2}(m+1)^{2}}\right) \\
& =\pi m(m+1)-\frac{\pi^{2}}{4} .
\end{aligned}
$$

This proves that for $m \geq 1, N(k) \geq\left\lceil\pi m(m+1)-\pi^{2} / 4\right\rceil$.

Observe that using standard trigonometric formulae [20], we get $f(n)=2 \sum_{i=1}^{m} \sin (i \pi / n)-$ $1=\frac{2 \sin ((m-1) \pi /(2 n)) \sin (m \pi /(2 n))}{\sin (\pi /(2 n))}-1$. Theorem 2 now immediately implies that the value of $N(k)$ can be computed by checking the sign of the function $f(n)$ at maximum $\left\lceil\pi^{2} / 4+1\right\rceil$ values of $n$. The minimum of value of $n$ at which the function $f$ is non-positive is the value of $N(k)$. Thus, we have the following theorem:

Corollary 1. For every odd positive integer $k=2 m+1(m \geq 1), N(k)$ can be computed in constant time. 


\section{Extensions of Theorem 1}

In this section we show that Theorem 1 can be extended to a larger family of point sets, which have one axis of symmetry. A set $S$ of $k=2 m+1(m \geq 1)$ points in the plane lying on the circumference of a unit circle with center at $o$, is said to be reflection symmetric if there exits a point $s_{0} \in S$ such that, for every point $s_{i} \in S \backslash\left\{s_{0}\right\}$, in the open halfplane containing $s_{i}$, there exists a point $s_{i}^{\prime} \in S \backslash\left\{s_{0}, s_{j}\right\}$, in the open halfplane not containing $s_{i}$, which is the reflection of the point $s_{i}$ about the line $o s_{0}$. The point $s_{0}$ will be called the pivot of $S$ and the line $o_{0}$ the line of symmetry of $S$. It is easy to see that a set of points lying on the vertices of a regular polygonal chain is reflection symmetric.

As any reflection symmetric point set is symmetric about its line of symmetry, the FermatWeber point of such a point set must lie on the line of symmetry. For a reflection symmetric point set $S$, with pivot at the point $s_{0}$, associate the rectangular coordinate system with origin at $o$ and the horizontal axis along the line $o s_{0}$. In this coordinate system, the coordinate of the point $s_{0}$ is $(1,0)$. Denote the points in $S$, as $s_{m}, \ldots, s_{2}, s_{1}, s_{0}, s_{1}^{\prime}, s_{2}^{\prime} \ldots, s_{m}^{\prime}$, with the points taken in the clockwise order, such that the point $s_{i}^{\prime}$ is the reflection of the point $s_{i}$ about the line $o s_{0}$, for each $i \in\{1,2, \ldots, m\}$ (Figure 3(a)). Let the coordinate of the point $s_{i}$ be $\left(\cos \theta_{i}, \sin \theta_{i}\right)$, where $0<\theta_{i}<\pi$. This implies that the coordinate of its reflection $s_{i}^{\prime}$ is $\left(\cos \theta_{i},-\sin \theta_{i}\right)$.

Let $p:=(x, 0)$ be any point on the $x$-axis. Now, for $i \in\{1,2, \ldots, m\}, d\left(s_{i}, p\right)=d\left(s_{i}^{\prime}, p\right)$, and the sum of distances of the elements in $S$ from a point $p$ is,

$$
\psi_{S}(x)=2 \sum_{i=1}^{m} d\left(s_{i}, p\right)+d\left(s_{0}, p\right)=2 \sum_{i=1}^{m} \sqrt{x^{2}-2 x \cos \theta_{i}+1}+(1-x) .
$$

Let $S$ be a reflection symmetric point set with pivot at the point $s_{0}$, and $\theta_{1}, \theta_{2}, \ldots, \theta_{m}$ be as described above. Such a set $S$ is said to satisfy Condition $A$ if $2 \sum_{i=1}^{m} \sin \left(\theta_{i} / 2\right)-1 \leq 0$. Now, it can be easily seen by following the proof of Lemma 1, that the Fermat-Weber point of $S$ coincides with the pivot $s_{0}$ if and only if the value of the function $\psi_{S}^{\prime}(x)=\frac{d}{d x} \psi_{S}(x)$ at $x=1$ is negative. Since $\psi_{S}^{\prime}(1)=2 \sum_{i=1}^{m} \sin \left(\theta_{i} / 2\right)-1$, we have the following theorem,

Theorem 3. For every odd positive integer $k=2 m+1(m \geq 1)$, the Fermat-Weber point of a reflection symmetric point set $S$, with $|S|=k$, coincides with the pivot of $S$, if and only if $S$ satisfies Condition A.

An interesting property and well-known property of the Fermat-Weber point of a set of non-collinear points is that the position of the Fermat-Weber point remains unchanged if the points in the set are moved along the rays joining them with the Fermat-Weber point of the set [14]. This fact can be applied to extend Theorem 3 to an even more general family of point set.

Let $S$ be a reflection symmetric point set with $|S|=k=2 m+1(m \geq 1)$ and $s_{0} \in S$ be the pivot. Define the extension of $S$ (to be denoted by $\mathcal{A}(S)$ ) as the set of all $k$ element point sets obtained by moving the points of $S \backslash\left\{s_{0}\right\}$ along the rays $\overrightarrow{s_{i} s_{0}}$, for $s_{i} \in S \backslash\left\{s_{0}\right\}$. It is clear that the point $s_{0}$ belongs to any set $T \in \mathcal{A}(S)$, and we call it the pivot point of the family $\mathcal{A}(S)$. The following result now follows immediately from Theorem 3 and the above discussion. 


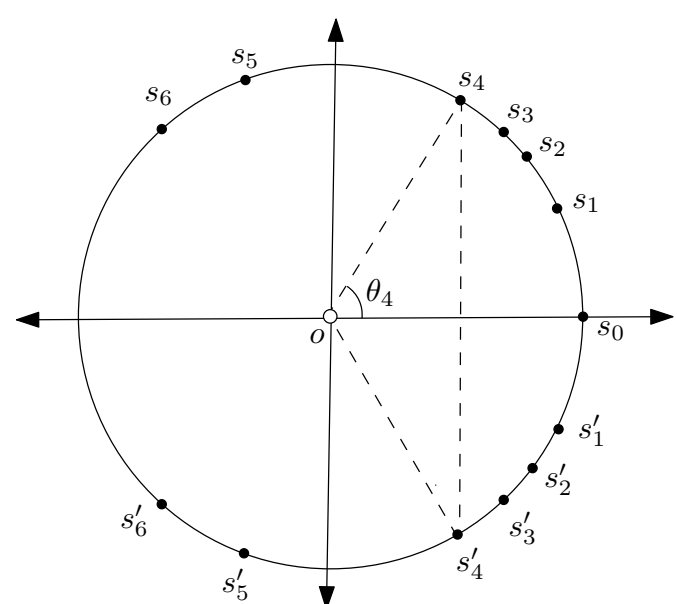

(a)

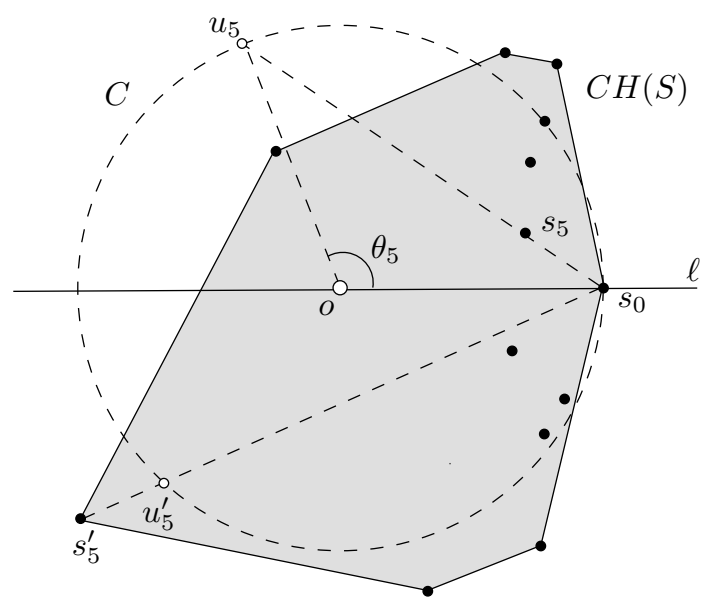

(b)

Fig. 3. (a) Reflection symmetric point set, (b) Illustration for the recognition algorithm.

Theorem 4. Let $S$ be a reflection symmetric point set with $|S|=k(k \geq 3)$ and pivot at the point $s_{0}$. The Fermat-Weber point of any set $T \in \mathcal{A}(S)$ coincides with the pivot point $s_{0}$ if and only if the Fermat-Weber point of $S$ coincides with $s_{0}$, that is, if and only if $S$ satisfies Condition A.

We now present a simple algorithm which recognizes whether a given point set belongs to the extension of a reflection symmetric point set. If it does, the algorithm also determines whether the Fermat-Weber point of such a set coincides with its pivot, by verifying Condition A.

Given a point set $Z$, we denote by $C H(Z)$ the convex hull of $Z$, and by $|C H(Z)|$ the number of vertices of $Z$ in $C H(Z)$.

Theorem 5. Given a point set T, with $|T|=k=2 m+1(m \geq 1)$ and $|C H(T)|=h$, there exists an $O(h k \log k)$ time algorithm which determines whether $T$ belongs to the extension of some reflection symmetric point set $S$, and whether the Fermat-Weber point of $T$ coincides with the pivot point of $\mathcal{A}(S)$.

Proof. Observe that if $T$ belongs to the extension of some set $S$, then the pivot point of the family $\mathcal{A}(S)$ lies on the convex hull of $S$. Further, a point $s_{0}$ on the convex hull of $T$ will be the pivot of some reflection symmetric point set if and only if the angle bisectors of the $m$ angles $\angle s_{i} s_{0} s_{i}^{\prime}, i=1,2, \ldots, m$ coincide, where $s_{1}, s_{2}, \ldots, s_{m}, s_{m}^{\prime}, \ldots, s_{2}^{\prime}, s_{1}^{\prime}$ are the points of $T \backslash\left\{s_{0}\right\}$ ordered by radially sorting them about $s_{0}$ in the counterclockwise direction.

We begin by finding out the convex hull of $S$ which requires $O(k \log h)$ time [717]. For every point in $C H(T)$, we radially sort the remaining points of $T$ about that point, and check whether the angle bisector of the $m$ angles described above coincide. The radial sorting step requires $O(k \log k)$ time after which the $m$ bisectors can be checked in $O(k)$ time. Since this has to be done for all the vertices of the convex hull of $T$, the total running time of the algorithm is $O(h k \log k)$.

Let $S$ be the reflection symmetric point set such that $T \in \mathcal{A}(S)$. The set $S$ can now be constructed from $T$ in $O(k)$ time as follows: The point $s_{0}$ identified above will clearly be 
the pivot point of $\mathcal{A}(S)$. Let $l$ be the common angle bisector of the $m$ angles described above (Figure 3(b)). Construct the unit circle $C$ with center $o$ on the line $\ell$ and passing through the the point $s_{0}$. For every point $s_{i} \in T \backslash\left\{s_{0}\right\}$, let $u_{i}$ be the point where the ray $\overrightarrow{s_{i} s_{0}}$ intersects the circumference of $C$ (Figure 2(b)). (Note that if some point $s_{i} \in T$ lies on the circumference of $C$, then $u_{i}=s_{i}$.) If $S_{0}=\left\{u_{i} \mid s_{i} \in S \backslash\left\{s_{0}\right\}\right\}$, then $S=S_{0} \cup\left\{s_{0}\right\}$. Condition $A$ for the point set $S$ can now be checked in $O(k)$ time and the result follows.

\section{Conclusions}

In this paper, we have explored the geometric properties of the Fermat-Weber point of polygonal chains. From the uniqueness of the Fermat-Weber point it is known that the Fermat-Weber point of a regular $n$-gon coincides with its circumcenter. However, when some vertices of the regular polygon are missing, the Fermat-Weber point can no longer be predicted exactly. Here, we show that Fermat-Weber point of polygonal chains, which are obtained by deleting a set of consecutive vertices of a regular polygon, can be predicted exactly in some situations. We show that for every odd positive integer $k$, there exists a smallest integer $N(k)$ such that for all $n \geq N(k), \mathcal{W}\left(C_{n}(k)\right)$ coincides with the root vertex of $C_{n}(k)$. This interesting geometric result can be thought of as an extension of the complementary problem of Courant and Robbins'. We also extend our results to more a general class point set and give a simple $O(h k \log k)$ time algorithm for identifying whether a given set of $k$ points, with $|C H(S)|=h$, belongs to such a class.

It may be interesting to find generalizations of this result to higher dimensions and to more general distance functions. However, as mentioned by Plastria [21], there does not seem to be much hope in geometrically predicting the Fermat-Weber point unless the given point set has a highly symmetric arrangement.

Acknowledgement. The author wishes to thank Prof. Probal Chaudhuri of Indian Statistical Institute, Kolkata for his insightful suggestions and for his inspirational guidance. The constructive comments of the anonymous reviewer are also thankfully acknowledged. The author also thanks Wolfram Research for inviting the work to be posted as a live demo in the Wolfram Demonstration Project.

\section{References}

1. A. K. Abu-Affash, M. J. Katz, Improved bounds on the average distance to the Fermat-Weber center of a convex object, Information Processing Letters, Vol. 109 (6), 329-333, 2009.

2. L. Anderegg, M. Cieliebak, G. Prencipe, Efficient Algorithms for Detecting Regular Point Configurations; Lecture Notes in Computer Science, Vol. 3701, 23-35, 2005.

3. C. Bajaj, The Algebraic Degree of Geometric Optimization Problems; Discrete and Computational Geometry, Vol. 3, 177-191, 1988.

4. P. Bose, A. Maheshwari, P. Morin, Fast Approximations for Sums of Distances, Clustering and the Fermat-Weber Problem; Computational Geometry: Theory and Applications, Vol. 24, 135-146, 2003.

5. R. E. Burkard, M. Galavii, E. Gassner, The inverse Fermat-Weber problem; European Journal of Operational Research Vol. 206, 11-17, 2010.

6. P. Carmi, S. Har-Peled, M. J. Katz: On the Fermat-Weber center of a convex object, Computational Geometry: Theory and Applications, Vol. 32,188-195, 2005.

7. T. M. Chan, Output-sensitive results on convex hulls, extreme points, and related problems; Discrete and Computational Geometry, Vol. 16, 369-387, 1996. 
8. P. Chaudhuri, On a Geometric Notion of Quantiles for Multivariate Data; Journal of the Amercian Statistical Association, Vol. 91, 862-872, 1996.

9. E. Cockayne, Z. Melzak, Euclidean Constructibility in Graph-Minimization Problems; Math. Magazine, Vol. 42, 206-208, 1969.

10. Z. Drezner, K. Klamroth, A. Schöbel, G. O. Wesolowsky, The Weber Problem, in Z. Drezner, H. W. Hamacher (Eds.), Facility Location: Applications and Theory; Springer, 2002.

11. A. Dumitrescu, M. Jiang, C. D. Toth, New bounds on the average distance from the Fermat-Weber center of a planar convex body, Discrete Optimization Vol. 8 (3), 417-427, 2011.

12. A. Dumitrescu, C. D. Toth, G. Xu, On stars and Steiner stars, Discrete Optimization, Vol. 6 (3), 324-332, 2009.

13. G. Fagnano, Problemata quaedam ad methodum maximorum et minimorum spectantia; Nova Acta Eruditorum, 281-303, 1775 (Mensis Iunii, published in 1779).

14. C. Gini, L. Galvani, Di talune estensioni dei concetti di media a caratteri qualitivi; Metron, Vol. 8., 3-209, 1929. (Partial English Translation in Journal of the Amercian Statistical Association, Vol. 25, 448-450.)

15. J. B. S. Haldane, Note on the Median of a Multivariate Distribution; Biometrika, Vol. 35, 414-415, 1948.

16. G. Jalal, J. Krarup, Geometrical Solution to the Fermat Problem with Arbitrary Weights; Annals of Operations Research Vol. 123, 67-104, 2003.

17. D. G. Kirkpatrick, R. Seidel, The utimate planar convex hull algorithm?; SIAM Journal of Computing, Vol. 15, 287-299, 1986.

18. J. Krarup, S. Vajda, On Torricelli's Geometrical Solution to a Problem of Fermat; IMA Journal of Mathematics Applied in Business and Industry Vol. 8, 215-224, 1997.

19. Y. S. Kupitz, H. Martini, Geometric Aspects of the Generalized Fermat-Weber Problem; Intuitive Geometry, Bolyai Soceity, Mathematical Studies 6, 55-127, 1997.

20. S. L. Loney, Plane Trigonometry, Part I; Cambridge University Press, London, 1966.

21. F. Plastria, Four Point Fermat Location Problems Revisited : New Proofs and Extensions of Old Results; IMA Journal of Management Mathematics, Vol. 17, 387-396, 2006.

22. E. Torricelli (c. 1640), De maximis et minimis; Opere di Evangelista Torricelli, G. Loria and G. Vassura (Eds.), Faenza, Italy, 1919.

23. Y. Vardi, C.H. Zhang, A Modified Weiszfeld Algorithm for the Fermat-Weber Location Problem; Math. Program., Ser. A, Vol. 90, 559-566, 2001.

24. A. Weber, Uber Den Standord Der Industrien, Tubigen, 1909. (English Translation by C. J. Freidrich, Alfred Weber's Theory of Location of Industries; Chicago University Press, 1929.)

25. E. Weiszfeld, Sur le point pour lequel la somme des distances de $n$ points donnes est minimum; Tohoku Mathematics Journal, Vol. 43, 355-386, 1937. (Translated and annotated by F. Plastria, On the point for which the sum of distances to $n$ given points is minimum; Annals of Operations Research, Vol. 167 (1), 7-41, 2009.)

26. G. Wesolowsky, The Weber problem: History and Perspective; Location Science, Vol. 1, 5-23, 1993.

27. I.M. Yaglom, Geometric Transformations I; Random House, New York, 1962, (Translated from Russian by Allen Shields). 